\title{
Brain glycogen decreases during prolonged exercise
}

\author{
Takashi Matsui ${ }^{1,4}$, Shingo Soya ${ }^{1}$, Masahiro Okamoto ${ }^{1,4}$, Yukio \\ Ichitani $^{2}$, Kentaro Kawanaka ${ }^{3}$ and Hideaki Soya ${ }^{1}$ \\ ${ }^{1}$ Laboratory of Exercise Biochemistry and Neuroendocrinology, \\ ${ }^{2}$ Department of Behavioral Neuroscience, Institute of Psychology, University of Tsukuba \\ Graduate School of Comprehensive Human Sciences, 1-1-1 Tennoudai, Tsukuba, \\ Ibaraki 305-8574, Japan \\ ${ }^{3}$ Department of Health and Nutrition, Niigata University of Health and Welfare, 1398 \\ Shimami-cho, Kita-ku, Niigata, Niigata 950-3198, Japan \\ ${ }^{4}$ Research Fellow of the Japan Society for the Promotion of Science, 8 Ichiban-cho, \\ Chiyoda-ku, Tokyo 102-0082, Japan
}

Running title: Brain glycogen reduction and hypoglycemia during prolonged exercise Three Key words: Prolonged exercise, Hypoglycemia, Brain glycogen

Total number of words: 4,485 words

\section{Corresponding author:}

Hideaki Soya, Ph.D.

Laboratory of Exercise Biochemistry and Neuroendocrinology, University of Tsukuba Graduate School of Comprehensive Human Sciences, 1-1-1 Tennoudai, Tsukuba 305-8574, Ibaraki, Japan. E-mail: hsoya@taiiku.tsukuba.ac.jp 


\section{Non-technical summary}

Energy sources for the brain include not only blood glucose, but also astrocytic glycogen, especially when the blood-born glucose supply is shortage (e.g. hypoglycemia). Although untested, it is hypothesized that during prolonged exercise that induces hypoglycemia, the resultant hypoglycemia may cause a decrease in brain glycogen. Here, we tested this hypothesis, and provide evidence that brain glycogen decreases during prolonged exercise with hypoglycemia. Furthermore, in the cortex, we show that the brain glycogen decrease during prolonged exercise is associated with activation of monoamine metabolism, which could be a factor inducing central fatigue. Since the discovery of muscle glycogen depletion as a candidate of peripheral fatigue during prolonged exercise, this is the first study to show that brain glycogen can decrease by prolonged exercise. These findings may provide a clue towards understanding the mechanisms related to central fatigue. 


\section{Abstract}

Brain glycogen could be a critical energy source for brain activity when the glucose supply from the blood is inadequate (hypoglycemia). Although untested, it is hypothesized that during prolonged exhaustive exercise that induces hypoglycemia and muscular glycogen depletion, the resultant hypoglycemia may cause a decrease in brain glycogen. Here, we tested this hypothesis and also investigated the possible involvement of brain monoamines with the reduced levels of brain glycogen. For this purpose, we exercised male Wistar rats by treadmill for different durations (30-120 min) at moderate intensity $(20 \mathrm{~m} / \mathrm{min})$ and measured brain glycogen levels of rats using the method of high-power microwave irradiation $(10 \mathrm{~kW})$. At the end of 30 and $60 \mathrm{~min}$ of running, the brain glycogen levels remained unchanged from resting levels, but liver and muscle glycogen decreased. After $120 \mathrm{~min}$ of running, the glycogen levels decreased significantly by $37 \sim 60 \%$ in five discrete brain loci (the cerebellum $60 \%$, cortex $48 \%$, hippocampus $43 \%$, medulla oblongata $37 \%$, and hypothalamus $34 \%$ ) compared to those of the sedentary control. The brain glycogen levels in all five regions after running were positively correlated with the respective blood and brain glucose levels. Further, in the cortex, the levels of methoxyhydroxyphenylglycol (MHPG) and 5-hydroxyindoleacetic acid (5-HIAA) with potential involvement in degradation of the brain glycogen increased during prolonged exercise and negatively correlated with the glycogen levels. These results support the hypothesis that brain glycogen could decrease with prolonged exhaustive exercise. Increased monoamines together with hypoglycemia should be associated with the development of decreased brain glycogen, suggesting new clue towards the understanding of central fatigue during prolonged exercise.

\section{Abbreviations list:}

NA, noradrenalin; 5-HT, 5-hydroxytryptamine; MI, microwave irradiation; MHPG, methoxyhydroxyphenylglycol; 5-HIAA, 5-hydroxyindoleacetic acid; PCA, perchloric acid; HPLC, high-performance liquid chromatography 


\section{Introduction}

Glycogen could be an important energy source for the brain. In the brain, the glucose storage molecule glycogen is located entirely in astrocytes (Wender et al., 2000). Astrocytic glycogen is degraded to provide neuroprotection primarily under conditions that induce glucose deprivation such as hypoglycemia (Herzog et al., 2008). Furthermore, even under normal physiological conditions, astrocytic glycogen is degraded to support axonal function in response to sudden increases in energy demand during neurotransmission (Swanson et al., 1992). Astrocytic glycogen would be broken down into lactate, as fuel for activated neurons, via increased noradrenalin (NA), histamine, 5-hydroxytryptamine (5-HT), and vasoactive intestinal peptide (Benington \& Heller, 1995; Brown, 2004).

Physical exercise impacts nearly every system of the body, including muscles and the brain (Secher et al., 2008); thus the energy supply available from immediate reserve sources for these organs plays a crucial role in maintaining their increased functions. During exercise, muscle glycogen decreases in an activity-dependent manner (exercise intensity and/or duration), and in turn supplies energy for muscle activity (Gollnick et al., 1974). Furthermore, during prolonged exhaustive exercise is performed, rats become hypoglycemic with marked depletion in muscular and liver glycogen at exhaustion (Winder et al., 1987). Although the brain increases glucose utilization as an energy source during exercise (Vissing et al., 1996), it remains unknown whether glycogen is utilized in the brain as an energy source during exercise.

Prolonged exercise increases monoamines (i.e. NA and 5-HT) levels in the brain (Newsholme et al., 1992; Pagliari \& Peyrin, 1995). Moreover, brain glycogen breakdown occurs during exercise via a $\beta$-adrenergic receptor-mediated mechanism as shown by measuring the concentration differences in arterial-jugular venous glucose, lactate, and oxygen, and their ratios (Ide et al., 2000; Larsen et al., 2008). Based on these findings, it has been hypothesized that exercise induces depletion of brain glycogen through hypoglycemia and activation of monoamines metabolism in the brain (Nybo \& Secher, 2004).

This hypothesis, however, remains to be tested because of technical difficulties in determining levels of brain glycogen during exercise with accuracy and precision. Indeed, brain glycogen is rapidly depleted by the activation of glycogenolysis 
enzymes under hypoxic-ischemic conditions that occur after the animals are sacrificed. Postmortem instability of glycogen in the brain prevents traditional biochemical methods of measurement (Kong et al., 2002). Therefore, we first established an accurate method for determining brain glycogen levels using high-power $(10 \mathrm{~kW})$, focused microwave irradiation (MI) as a gold standard, which momentarily inactivates glycogenolytic and glycosynthetic enzymes. Furthermore, we designed experiments to examine the effects of exercise on glycogen levels in different brain loci along with checking the involvement of blood glucose and monoamines levels using $\mathrm{MI}$, which allowed us to test the hypothesis that exercise induces brain glycogen depletion through increased monoamines and under hypoglycemic conditions. Our results have shown, for the first time, that exercise results in a decrease in brain glycogen due to hypoglycemia and to an increase of NA and 5-HT metabolism in the cortex.

\section{Methods}

The present study consisted of four major steps. In Pilot Study 1, we examined brain glycogen levels with and without anesthesia, in order to determine valid conditions for analyzing brain-glycogen levels. In Pilot Study 2, we also checked the validity of brain glycogen determination, examining insulin-induced hypoglycemia on cerebellum glycogen levels (Herzog et al., 2008). Then, the effects of prolonged exercise on glycogen levels in nine discrete brain loci (third step) and the effects of various durations of exercise on glycogen levels in five brain loci (forth step) were examined respectively. Details of Pilot Studies 1 and 2 are presented in the Supplement (available in the online version of this article).

\section{Materials}

All chemicals, including amyloglucosidase, hexokinase, NADP+-dependent glucose-6-phosphate dehydrogenase, NADP+, ATP, EDTA, MgSO4, glucose, glucose-6-phosphate, $\mathrm{KOH}$, imidazole, perchloric acid, and Tris- $\mathrm{HCl}$, were purchased from Sigma (St. Louis, MO, USA) and Nacalai tesque (Kyoto, Japan).

Adult male Wistar rats (250 - 270 g) (SLC Inc., Shizuoka, Japan) were housed and cared for in an animal facility, fed a standard pellet diet (MF, Oriental Yeast Co., Ltd, 
Ibaraki, Japan), and given water ad libitum. The room temperature was maintained between $22^{\circ}$ and $24^{\circ} \mathrm{C}$ under a 12-hour light:12-hour dark cycle (lights on: 7:00-19:00). All experimental protocols were conducted in accordance with University of Tsukuba Animal Experiment Committee guidelines. Our experiments comply with the policies and regulations of The Journal of Physiology (Drummond, 2009).

\section{Habituation to treadmill running}

After a 1-wk acclimatization period, rats were habituated to run on a treadmill (SN-460, Shinano, Tokyo, Japan) for a total of five sessions over six days. The running duration was $30 \mathrm{~min} /$ day, and the running speed was gradually increased from 5 to 25 $\mathrm{m} / \mathrm{min}$ (Nishijima \& Soya, 2006).

\section{Surgery}

Surgery was performed according to methods described by Soya et al. (2007a). After habituation to treadmill running, the rats were anesthetized with sodium pentobarbital (50 mg/kg, i.p.), and a silicone catheter was inserted into the jugular vein and fixed with a silk thread $(32 \mathrm{~mm})$. The external distal end of the catheter was fixed at the animal's nape.

\section{Prolonged exhaustive exercise}

Two days after the habituation period, rats were fasted before exercise for 2 hours to obtain stable glycogen levels in the brain, and exercised by treadmill running at $20 \mathrm{~m} / \mathrm{min}$ for $120 \mathrm{~min}$ ( $\mathrm{n}=5-11$ rats). A sedentary group of animals ( $\mathrm{n}=11$ rats) was placed on a stationary treadmill for $120 \mathrm{~min}$. Fatigue during prolonged exercise of an intensity between 65 and $85 \%$ maximum oxygen uptake $\left(\mathrm{V}_{2} \max \right)$ are associated with glycogen depletion in active skeletal muscles (Gollnick et al., 1974). Brooks \& White (1978) and Soya et al. (2007a) have reported that running at $20 \mathrm{~m} / \mathrm{min}$ on the treadmill is equivalent to $50-70 \% \mathrm{~V}_{2 \max }$ for rats. Thus, prolonged exercise at moderate intensity in this study should be sufficient to induce depletion of glycogen in active muscles, warranting its validity as exhaustive exercise.

\section{Exercise of various time durations}

Two days after the habituation period, rats fasted for 3 hours, and ran on a 
treadmill at $20 \mathrm{~m} / \mathrm{min}$ for 0 (pre-exercise), 30, 60, or $120 \mathrm{~min}(\mathrm{n}=5-6$ rats per group).

\section{Tissue preparation}

Immediately after exercise or rest, rats were anesthetized with isoflurane (a mixture of $30 \%$ vol/vol isoflurane in propylene glycol, Dainippon Sumitomo Pharma Co., Ltd., Osaka, Japan) in a bell jar and sacrificed using focused microwave irradiation (MI) (NJE-2603, New Japan Radio Co., Ltd., Tokyo, Japan; 10 kW, $1.2 \mathrm{sec}$ ). Following the MI, nine brain loci (cortex, septum, striatum, hippocampus, thalamus, hypothalamus, midbrain, cerebellum, and medulla oblongata) were collected using a method modified from Hirano et al. (2006). Two skeletal muscles (soleus and plantaris), the liver, and blood samples were also collected. All specimens were stored at $-80^{\circ} \mathrm{C}$ for subsequent biochemical analysis.

\section{Blood glucose and lactate assays}

Blood lactate and glucose were measured using an automated glucose-lactate analyzer (2300 Stat Plus, Yellow Springs Instruments, USA).

\section{Brain glucose and glycogen assays}

Glucose and glycogen measurements were taken according to methods described by Kong et al. (2002). Tissues were homogenized (Polytron, Kinematica, Kriens-Luzern, Switzerland; setting 6; $60 \mathrm{sec}$ ) in ice-cold $6 \%$ perchloric acid (PCA) containing $1 \mathrm{mM}$ EDTA. For tissue glycogen content measurements, glycogen was hydrolyzed to glucose in $100-\mu l$ aliquots of homogenate that were removed and incubated for $1 \mathrm{hr}$ at $37^{\circ} \mathrm{C}$ with $1 \mathrm{ml}$ of $0.2 \mathrm{M}$ sodium acetate, $20 \mu \mathrm{l}$ of $1.0 \mathrm{M} \mathrm{KHCO}$, and $20 \mathrm{U} / \mathrm{ml}$ of amyloglucosidase. The addition of $0.5 \mathrm{ml}$ of PCA solution stopped the reaction. After centrifugation $\left(14,000 \mathrm{~g}\right.$ for $10 \mathrm{~min}$ at $\left.4^{\circ} \mathrm{C}\right)$, supernatants were neutralized with a $\mathrm{KOH}$ solution consisting of (in $\mathrm{M}$ ): $3 \mathrm{KOH}, 0.3$ imidazole, and $0.4 \mathrm{KCl}$. The supernatants were then centrifuged $\left(16,000 \mathrm{~g}\right.$ for $10 \mathrm{~min}$ at $\left.4^{\circ} \mathrm{C}\right)$ and assayed for glucose content. To measure endogenous (background) glucose levels, non-hydrolyzed samples were obtained by centrifuging the homogenates $\left(14,000 \mathrm{~g}\right.$ for $10 \mathrm{~min}$ at $\left.4^{\circ} \mathrm{C}\right)$ and adjusting the $\mathrm{pH}$ of the supernatants to a final level of 6-8 with $\mathrm{KOH}$ solution. Neutralized samples were mixed thoroughly, centrifuged $\left(16,000 \mathrm{~g}\right.$ for $10 \mathrm{~min}$ at $\left.4^{\circ} \mathrm{C}\right)$, and assayed for endogenous glucose levels. The glucose content assay was performed 
in 96-well plates using a coupled enzyme assay method modified from a previous study (Passonneau \& Lauderdale, 1974). A total of $200 \mu$ of a reaction solution containing 50 $\mathrm{mM}$ Tris- $\mathrm{HCl}(\mathrm{pH} 8.1), 0.5 \mathrm{mM}$ ATP, $0.5 \mathrm{mM} \mathrm{NADP+,} 5 \mathrm{mM} \mathrm{MgSO}$, and $0.1 \mathrm{U} / \mathrm{ml}$ glucose-6-phosphate dehydrogenase was added to each well. The plate was then placed in a fluorescence plate reader (Arvo, Perkin Elmer, Groningen, Netherlands) and shaken, and measurements of the resultant NADPH were taken at $350 \mathrm{~nm}$ excitation and $450 \mathrm{~nm}$ emission. After the addition of hexokinase $(0.3 \mathrm{U})$ to each well, plates were shaken, and measurements were taken after a 30 min incubation period. Tissue glycogen levels, indicated as glucose units, were calculated by subtracting the final micromolar concentration of glucose per gram of wet weight of the non-hydrolyzed tissue sample from the micromolar concentration of glucose per gram of wet weight of the hydrolyzed tissue sample. Our samples showed the averaged hypothalamic glucose levels of sedentary rats were $2.8 \mu \mathrm{mol} / \mathrm{g}$ (baseline levels), which correspond well with previous findings from microdialysis studies of extracellular fluid in the ventromedial nucleus of the hypothalamus (de Vries et al., 2003). It was the same in the hypoglycemic levels induced by insulin (Herzog et al., 2008). As for the brain glycogen levels indicating methodological validity, sedentary control levels of glycogen in the hippocampus and cerebellum were approximately $10 \mu \mathrm{mol} / \mathrm{g}$ tissue (Figure 1D), which is consistent with previous findings using high-power MI (9.5 kW, $1.7 \mathrm{sec} ; 10 \mathrm{~kW}, 1.2$ sec) (Kong et al., 2002; Herzog et al., 2008).

\section{Brain lactate assays}

Lactate measurements were taken according to the method described by Passonneau and Lauderdale (1974). Our average hypothalamic lactate levels of sedentary animals were $1.1 \mu \mathrm{mol} / \mathrm{g}$, which corresponds well with previous findings from microdialysis studies of extracellular fluid in the hypothalamus (Wang et al., 2002), indicating methodological validity.

\section{Monoamine assays}

Measurements of monoamines, NA, 5-HT and dopamine (DA), and their metabolites, MHPG, 5-HIAA and DOPAC, respectively, were taken using high-performance liquid chromatography (HPLC) with electrochemical detection according to methods described by Takeda et al. (1990). Briefly, $0.5 \mathrm{ml}$ of a $0.05 \mathrm{M}$ PCA 
solution containing $2.0 \mathrm{mM}$ sodium bisulfate and $0.02 \mathrm{mM}$ EDTA $2 \mathrm{Na}$ was added to the samples of cortical tissue. After homogenization followed by centrifugation at $12,000 \mathrm{~g}$ for $5 \mathrm{~min}$, the supernatants were used to determine the levels of each of the above molecules after filtration through a $0.45 \mu \mathrm{m}$ filter (Millipore, MA, USA). An LC/ECD (electron capture dissociation) system equipped with an electrochemical detector (Shimazu, Japan) was used for all measurements. The detector conditions were as follows: guard cell potential, E, $450 \mathrm{mV}$; analytical cell potentials, E1, -50 mV and E2, $450 \mathrm{mV}$; and sensitivity, $2 \mu \mathrm{A}$. Separation was carried out using a 5- $\mu \mathrm{m}$ MCM C18 (4.6 $\varphi \times 150 \mathrm{~mm}$; MC) column at $20^{\circ} \mathrm{C}$ under isocratic conditions with a mobile phase of acetonitrile, methanol, and $0.1 \mathrm{M}$ phosphate buffer adjusted to $\mathrm{pH} 3.0$ with phosphoric acid containing $0.05 \mathrm{M}$ citrate, $4.0 \mathrm{mM}$ sodium 1-heptasulfonate, and $0.1 \mathrm{mM}$ EDTA $2 \mathrm{Na}$. The flow rate and injection volume were set to $1.0 \mathrm{ml} / \mathrm{min}$ and $30 \mu \mathrm{l}$, respectively. A $30-\mu \mathrm{l}$ aliquot of $1,000 \mathrm{ng} / \mathrm{ml}$ isoproterenol hydrochloride was added to each sample as an internal standard, and $50 \mu$ of the same solution was used to wash the injection syringe. The concentrations of monoamines and their metabolites in brain tissue were determined based on their chromatographic peak heights relative to that of the internal standard (isoproterenol) using appropriate calibration curves in the Chromeleon software (Dionex, CA, USA).

\section{Statistics}

Data are expressed as mean \pm standard error (SEM) and were analyzed using Prism 5 (MDF Co., Ltd., Tokyo, Japan). Comparisons of each exercise group versus the sedentary group were performed using Student's t-test for unpaired data. Group comparisons were performed using a one-way ANOVA with Dunnett's or Tukey's post hoc tests. Simple correlations were calculated using Pearson's product-moment correlations. Statistical significance was assumed at $p$-values $<0.05$.

\section{Results}

\section{Brain glycogen levels after exercise}

The blood glucose level was $45 \%$ lower in the exercise group as compared to the sedentary group $(F=16.65 ; p<0.0001 ; F i g .1 A)$. The glycogen levels in the liver and 
skeletal muscles (slow type, m. soleus; fast type, m. plantaris) were found to be depleted by exercise (Fig. 1B, C). Moreover, glycogen levels decreased significantly by $37 \sim 60 \%$ with exercise in five discrete brains loci: the cerebellum $60 \%$, cortex $48 \%$, hippocampus 43\%, medulla oblongata 37\%, and hypothalamus 34\% ( $p<0.05$; Fig. 1D, E). Conversely, glycogen levels in the septum, midbrain, thalamus, and striatum were unchanged by exercise (Fig. 1D, E).

\section{Spatio-tepmporal changes in brain glycogen levels during exercise}

At the end of 30 and 60 min of running, blood glucose and lactate levels were unchanged compared to pre-exercise levels. At the end of 120 min of running, however, blood glucose was $46 \%$ lower, and blood lactate was five-fold higher than before exercise and under sedentary conditions ${ }^{\S}$ at $120 \mathrm{~min}\left(p<0.01\right.$; Fig. $2 \mathrm{~A}$; ${ }^{\S}$, data not shown). Similar to blood glucose levels, after 30 and 60 min of running, glycogen levels in the cortex, hippocampus, hypothalamus, cerebellum, and medulla oblongata did not significantly decrease from those before exercise and sedentary conditions $\$$ at $120 \mathrm{~min}$ however, they did decrease after 120 min of exercise ( $p<0.05$; Fig. 2F-J; ${ }^{\S}$, data not shown). Glycogen levels in the skeletal muscles and liver were significantly decreased after 30 min of running and further decreased after 60 and 120 min of running (Fig. $2 \mathrm{C}-\mathrm{E}$ ), while those of the skeletal muscles and liver under sedentary conditions $\$$ at 120 min remained unchanged throughout experiments (Fig. 2C-E, §, data not shown).

Glucose levels in the cortex, hippocampus, hypothalamus, cerebellum, and medulla oblongata were significantly decreased, and lactate levels in the cortex, hippocampus, hypothalamus, cerebellum, and medulla oblongata were significantly increased following 120 min of exercise (Table 1). Furthermore, the decrease in brain glycogen levels was positively correlated with the decrease in blood $(r=0.55 \sim 0.70 ; p<$ 0.05 ; Fig. 3A) and brain ( $r=0.59 \sim 0.91 ; p<0.001$; Fig. 3B) glucose levels, and was negatively correlated with the increase in brain lactate levels excluding the hypothalamus $(r=-0.47 \sim-0.65 ; p<0.05$; Fig. $3 C)$.

\section{Cortical monoamine metabolic levels after exercise}

Figure 4A-F shows the levels of monoamines and their metabolites in the cortex for different exercise durations. Monoamines, NA, 5-HT, and DA levels in the cortex increased with exercise but were not significantly different from pre-exercise 
levels (Fig. A-C). After 60 min of running, MHPG levels in the cortex were increased from pre-exercise levels ( $p<0.05$; Fig. 4D) and increased further after $120 \mathrm{~min}$ of running ( $p<0.05$; Fig. 4D). 5-HIAA levels in the cortex after 60 and 120 min of running were increased from pre-exercise levels ( $p<0.05$; Fig. 4E). 3,4-dihydroxyphenylacetic acid (DOPAC) in the cortex increased with exercise but was not different from pre-exercise levels (Fig. 4F). Finally, the increases in cortical MHPG and 5-HIAA levels were negatively correlated with the decrease in cortical glycogen levels $(r=-0.66 \sim$ 0.68; p < 0.01; Fig. 4G, H).

\section{Discussion}

This is the first report testing the hypothesis that prolonged exhaustive exercise, which induces hypoglycemia and muscle glycogen depletion, will result in decreased levels of brain glycogen, the principal energy store in brain, using a high-power MI (10 kW) which nearly immediately fixes brains, rendering glycogen-producing and -metabolizing enzymes inactive. We thus determined levels of brain glycogen, which decreased significantly by approximately $50 \%$ in brains of rats that underwent 120 min of running, compared to those of sedentary control rats (Fig. 1D); the cerebellum $60 \%$, cortex $48 \%$, hippocampus $43 \%$, medulla oblongata $37 \%$, and hypothalamus $34 \%$. Further, changes in brain glycogen correlated well with changes in blood glucose levels, and negatively correlated with changes in brain lactate levels. These results support the hypothesis that brain glycogen could decrease with prolonged exhaustive exercise that induces hypoglycemia. Further, in the cortex, MHPG and 5-HIAA levels, which are metabolites of $\mathrm{NA}$ and $5-\mathrm{HT}$, increased during prolonged exercise, and negatively correlated with decreased cortical glycogen, and it should be possible that such a decrease in cortical glycogen is also associated with increased metabolism of NA and 5-HT in the cortex. These results suggest that increased metabolism of NA and 5-HT in the cortex, probably in the whole brain, together with hypoglycemia should be associated with the development of decreased brain glycogen should be a new clue towards our understanding of central fatigue.

An exhaustive run for 120 min decreased blood glucose and brain glycogen levels in the cortex, hippocampus, hypothalamus, cerebellum, and medulla oblongata. 
To examine the possibility that decreased brain glycogen is due to hypoglycemia, we examined the effects of various durations of exercise on blood glucose and brain glycogen levels. Blood glucose levels remained unchanged after 30 or 60 min of exercise, but decreased after prolonged exercise (Fig. 2A). Similar to blood glucose levels, glycogen levels in the cortex, hippocampus, hypothalamus, cerebellum, and medulla oblongata decreased only with $120 \mathrm{~min}$ of exhaustive exercise. At the same time, both depletion of liver glycogen and hypoglycemia occurred (Fig. 2C-E), which may in turn result in decreased brain glucose levels (50 67\% of sedentary control, Table 1). Indeed, brain-glucose levels were correlated with blood-glucose levels $(r=0.71 \sim 0.86$, data not shown). Then brain glycogen levels during exercise were positively correlated with blood and brain glucose levels (Fig. 3A, B). These results suggest that the decrease in brain glycogen elicited by exercise would be due to hypoglycemia.

We found that brain lactate levels increased with prolonged exercise (Table 1) and brain glycogen levels during exercise were negatively correlated with brain lactate (Fig. 3C). It is thus likely that exercise-induced hypoglycemia causes astrocytic glycogenolysis, leading to the production of lactate as fuel for neurons in the brain. This is consistent with findings that an astrocyte-neuron lactate shuttle (Pellerin \& Magistretti, 1994; Brooks, 2009) and glycogen shunt (Shulman et al., 2001) function during exhaustive exercise. The increase in brain lactate would also be due to increased blood-lactate uptake by the brain (Ide et al., 2000). However, it is uncertain which is dominant, glycolysis or blood-lactate uptake, in the increase in brain lactate during exercise.

During exercise, skeletal muscle glycogen is degraded in an activity-dependent manner, exercise intensity and/or duration, and is utilized to supply energy for adenosine triphosphate (ATP) synthesis (Gollnick et al., 1974). Contrary Vissing et al. (1996) examined brain glucose uptake as an index of functional neuronal activity and found that glucose uptake was increased in motor and somatosensory cortices, and the cerebellum during $30 \mathrm{~min}$ of high-intensity running $(28 \mathrm{~m} / \mathrm{min}, \sim 85 \%$ of maximum oxygen consumption). Levels of c-Fos protein, as a cell activation marker, in the hypothalamus increase with moderate exercise just above the lactate threshold (LT); LT speed has been found to be around $20 \mathrm{~m} / \mathrm{min}$ in male rats who underwent 10-14 days of running habituation (Soya et al., 2007a). Furthermore, an increase of 
c-Fos protein levels in the brain stem (Ohiwa et al., 2006), and an increase of BDNF mRNA/protein levels in the hippocampus (Soya et al., 2007b). In the present study, a prolonged running test was performed at the LT level $(20 \mathrm{~m} / \mathrm{min})$, thus neuronal activity would be increased in the cortex, hippocampus, hypothalamus, cerebellum, and medulla oblongata. Therefore, brain glycogen decreases with prolonged exercise due to enhanced glycogenolysis, since astrocytic glycogenolysis is enhanced with neuro-activating stimulation (for example, sleep deprivation and sensory stimulation) (Swanson et al., 1992; Cruz \& Dienel, 2002; Kong et al., 2002).

The levels of MHPG and 5-HIAA, which are metabolites of NA and 5-HT, respectively, increased in the cortex during exercise, especially at 120 min (Figure 4). This is consistent with NA or $5-\mathrm{HT}$ levels increased in the cortex with prolonged (1 2 hours) treadmill exercise (Pagliari \& Peyrin, 1995; Gomez-Merino et al., 2001). Furthermore, as shown in Figures $4 \mathrm{G}$ and $4 \mathrm{H}$, the decrease in cortical glycogen levels during exercise was negatively correlated with increased cortical MHPG and 5-HIAA levels. Since brain glycogenolysis is promoted by activation of noradrenergic and/or serotonergic mechanisms (Benington \& Heller, 1995; Pachmerhiwala et al., 2010), our present results suggest that the reduction of cortex glycogen during exercise is not only due to hypoglycemia but also to increased NA and 5-HT metabolism. In addition, since neuronal activation would promote NA and 5-HT metabolisms, our results suggest that cortical neuronal activity increases with exercise. Thus, brain glycogenolysis could be promoted in order to supply ATP for neuronal activation during exercise.

Hypoglycemia and increased 5-HT levels in the brain could serve as indicators of central fatigue during prolonged exercise (Newsholme et al., 1992; Nybo \& Secher, 2004). On the other hand, hypoglycemia and 5-HT are factors of glycogenolysis in the brain (Benington \& Heller, 1995). Thus, brain glycogen decreases with prolonged exercise lead us to postulate that hypoglycemia together with brain activation with NA and/or 5-HT metabolism might be involved in the development of decreases in brain glycogen, and may shed light on studies examining how brain-glycogen metabolism is involved in the central fatigue during exercise (i.e., understanding how enhancing the effects of brain glycogen storage and availability may delay exercise-induced exhaustion).

Collectively, this study provides evidence indicating that exercise results in a decrease in brain glycogen due to hypoglycemia and to an increase of NA and 5-HT 
metabolism in the cortex. Exercise-induced hypoglycemia and increased 5-HT levels could be indicators of central fatigue; however, our findings suggest that whether these phenomena cause exhaustion depends on the reduction in brain-glycogen levels. These findings may aid in elucidating the mechanisms underlying central fatigue.

\section{Methodological implication}

Although brain glycogen has a long history of being difficult to determine, there are still few reports showing its in vivo implications. This is because the rapid breakdown of brain glycogen, compared with that of glycogen in peripheral areas, makes it impossible to reliably measure stable brain glycogen levels. However, a breakthrough study by Kong et al. (2002) revealed that a higher power (10 kW, 1.2) MI, which immediately fixes brains, rendering glycogen-producing and -metabolizing enzymes inactive (with an averaged tempetature of $82 \pm 3^{\circ} \mathrm{C}$ in their study), can allow the determination of values ten-fold higher than without MI. They were able to finely decline brain glycogen levels, which varied between brain regions, in rats with sleep deprivation and hypoglycemia compared to respective controls. Similarly to our study, Herzog et al. (2008) also recently reported high, and valid, brain glycogen levels in hypoglycemic rats using high-powered $\mathrm{MI}(9.5 \mathrm{~kW}, 1.7 \mathrm{sec})$. High-powered $\mathrm{MI}$ is necessary in order to correctly detect original brain glycogen levels and should be taken as the gold standard. Thus, our findings using $\mathrm{MI}$ to show prolonged exercise-induced decrease in brain glycogen is compelling.

\section{Acknowledgments}

This work was supported in part by Grant-in-Aid for Challenging Exploratory Research of the Japan Society for the Promotion of Science (no.21650166), by Grant-in-Aid for JSPS Fellows (no.10J00513), by a grant from the Kozuki foundation for sports and education, and by the Ministry of Education, Culture, Sports, Science and Technology (MEXT) for the Body and Mind Integrated Sports Sciences Project (2010).

\section{Appendix A. Supplementary data}

Supplementary data associated with this article can be found in the online version. 


\section{Tables}

Table 1. Glucose and lactate levels in five brain loci immediately after treadmill running exercise for 0 (Pre-Exercise), 30, 60, and $120 \mathrm{~min}$ ( $\mu \mathrm{mol} / \mathrm{g}$ wet tissue).

\begin{tabular}{cccccc}
\hline Brain region & & $0 \mathrm{~min}$ & $30 \mathrm{~min}$ & $60 \mathrm{~min}$ & $120 \mathrm{~min}$ \\
\hline \multirow{2}{*}{ Cortex } & Glucose & $2.6 \pm 0.1$ & $3.3 \pm 0.1^{*}$ & $3.2 \pm 0.2$ & $1.3 \pm 0.4^{*}$ \\
\cline { 2 - 6 } & Lactate & $1.1 \pm 0.0$ & $1.9 \pm 0.3^{*}$ & $1.9 \pm 0.3$ & $2.5 \pm 0.2^{* * *}$ \\
\hline \multirow{2}{*}{ Hippocampus } & Glucose & $2.8 \pm 0.1$ & $3.4 \pm 0.1^{*}$ & $3.5 \pm 0.1^{*}$ & $1.6 \pm 0.3^{*}$ \\
\cline { 2 - 6 } & Lactate & $1.3 \pm 0.0$ & $2.2 \pm 0.3$ & $2.0 \pm 0.3$ & $2.7 \pm 0.4^{* *}$ \\
\hline \multirow{2}{*}{ Hypothalamus } & Glucose & $2.8 \pm 0.1$ & $3.2 \pm 0.1$ & $3.0 \pm 0.1$ & $1.9 \pm 0.3^{*}$ \\
\cline { 2 - 6 } & Lactate & $1.1 \pm 0.1$ & $1.9 \pm 0.3^{*}$ & $1.8 \pm 0.3$ & $2.4 \pm 0.2^{* * *}$ \\
\hline \multirow{2}{*}{ Cerebellum } & Glucose & $3.1 \pm 0.1$ & $3.7 \pm 0.2$ & $3.4 \pm 0.1$ & $1.9 \pm 0.4^{*}$ \\
\cline { 2 - 6 } & Lactate & $0.9 \pm 0.1$ & $1.5 \pm 0.2^{*}$ & $1.5 \pm 0.2$ & $2.0 \pm 0.2^{* *}$ \\
\hline \multirow{2}{*}{ Medulla oblongata } & Glucose & $2.7 \pm 0.1$ & $3.2 \pm 0.1$ & $3.3 \pm 0.2$ & $1.6 \pm 0.3^{*}$ \\
\cline { 2 - 6 } & Lactate & $1.1 \pm 0.1$ & $1.9 \pm 0.3^{*}$ & $1.9 \pm 0.3$ & $2.5 \pm 0.2^{* * *}$ \\
\hline
\end{tabular}

Data are mean \pm SEM for $5-6$ rats. ${ }^{*}: p<0.05,{ }^{* * *}: p<0.0001$ vs 0 min (Dunnet's post hoc). 


\section{References}

Benington JH \& Heller HC (1995). Restoration of brain energy metabolism as the function of sleep. Prog Neurobiol 45, 347-360.

Brooks GA (2009). Cell-cell and intracellular lactate shuttles. J Physio/ 587, 5591-5600.

Brooks GA \& White TP (1978). Determination of metabolic and heart rate responses of rats to treadmill exercise. J Appl Physiol 45, 1009-1015.

Brown AM (2004). Brain glycogen re-awakened. J Neurochem 89, 537-552.

Cruz NF \& Dienel GA (2002). High glycogen levels in brains of rats with minimal environmental stimuli: implications for metabolic contributions of working astrocytes. J Cereb Blood Flow Metab 22, 1476-1489.

Gollnick PD, Piehl K \& Saltin B (1974). Selective glycogen depletion pattern in human muscle fibres after exercise of varying intensity and at varying pedalling rates. $J$ Physiol 241, 45-57.

Gomez-Merino D, Bequet F, Berthelot M, Chennaoui M \& Guezennec CY (2001). Site-dependent effects of an acute intensive exercise on extracellular 5-HT and 5-HIAA levels in rat brain. Neurosci Lett 301, 143-146.

Herzog RI, Chan O, Yu S, Dziura J, McNay EC \& Sherwin RS (2008). Effect of acute and recurrent hypoglycemia on changes in brain glycogen concentration. Endocrinology 149, 1499-1504.

Hirano M, Rakwal R, Shibato J, Agrawal GK, Jwa NS, Iwahashi H \& Masuo Y (2006). New protein extraction/solubilization protocol for gel-based proteomics of rat (female) whole brain and brain regions. Mol Cells 22, 119-125.

Ide K, Schmalbruch IK, Quistorff B, Horn A \& Secher NH (2000). Lactate, glucose and 
O2 uptake in human brain during recovery from maximal exercise. J Physiol 522, 159-164.

Kong J, Shepel PN, Holden CP, Mackiewicz M, Pack AI \& Geiger JD (2002). Brain glycogen decreases with increased periods of wakefulness: implications for homeostatic drive to sleep. J Neurosci 22, 5581-5587.

Larsen TS, Rasmussen P, Overgaard M, Secher NH \& Nielsen HB (2008). Non-selective beta-adrenergic blockade prevents reduction of the cerebral metabolic ratio during exhaustive exercise in humans. $J$ Physiol 586, 2807-2815.

Newsholme EA, Blomstrand E \& Ekblom B (1992). Physical and mental fatigue: metabolic mechanisms and importance of plasma amino acids. Br Med Bull 48, 477-495.

Nishijima T \& Soya H (2006). Evidence of functional hyperemia in the rat hippocampus during mild treadmill running. Neurosci Res 54, 186-191.

Nybo L \& Secher NH (2004). Cerebral perturbations provoked by prolonged exercise. Prog Neurobiol 72, 223-261.

Ohiwa N, Saito T, Chang H, Nakamura T \& Soya H (2006). Differential responsiveness of c-Fos expression in the rat medulla oblongata to different treadmill running speeds. Neurosci Res 54, 124-132.

Pachmerhiwala R, Bhide N, Straiko M \& Gudelsky GA (2010). Role of serotonin and/or norepinephrine in the MDMA-induced increase in extracellular glucose and glycogenolysis in the rat brain. Eur J Pharmacol 644, 67-72.

Pagliari R \& Peyrin L (1995). Norepinephrine release in the rat frontal cortex under treadmill exercise: a study with microdialysis. J Appl Physiol 78, 2121-2130. 
Passonneau JV \& Lauderdale VR (1974). A comparison of three methods of glycogen measurement in tissues. Anal Biochem 60, 405-412.

Pellerin L \& Magistretti PJ (1994). Glutamate uptake into astrocytes stimulates aerobic glycolysis: a mechanism coupling neuronal activity to glucose utilization. Proc Natl Acad Sci U S A 91, 10625-10629.

Secher NH, Seifert T \& Van Lieshout JJ (2008). Cerebral blood flow and metabolism during exercise: implications for fatigue. J Appl Physiol 104, 306-314.

Shulman RG, Hyder F \& Rothman DL (2001). Cerebral energetics and the glycogen shunt: neurochemical basis of functional imaging. Proc Natl Acad Sci U S A 98, 6417-6422.

Soya H, Mukai A, Deocaris CC, Ohiwa N, Chang H, Nishijima T, Fujikawa T, Togashi K \& Saito T (2007a). Threshold-like pattern of neuronal activation in the hypothalamus during treadmill running: establishment of a minimum running stress (MRS) rat model. Neurosci Res 58, 341-348.

Soya $H$, Nakamura $T$, Deocaris CC, Kimpara A, limura M, Fujikawa $T$, Chang $H$, McEwen BS \& Nishijima T (2007b). BDNF induction with mild exercise in the rat hippocampus. Biochem Biophys Res Commun 358, 961-967.

Swanson RA, Morton MM, Sagar SM \& Sharp FR (1992). Sensory stimulation induces local cerebral glycogenolysis: demonstration by autoradiography. Neuroscience $51,451-461$.

Takeda H, Matsumiya T \& Shibuya T (1990). Detection and identification modes for the highly sensitive and simultaneous determination of various biogenic amines by coulometric high-performance liquid chromatography. J Chromatogr 515, 265-278.

Vissing J, Andersen M \& Diemer NH (1996). Exercise-induced changes in local cerebral 
glucose utilization in the rat. J Cereb Blood Flow Metab 16, 729-736.

Wang L, Dong Y, Yu X, Shangguan DH, Zhao R, Han HW \& Liu GQ (2002). Analysis of glucose and lactate in dialysate from hypothalamus of rats after exhausting swimming using microdialysis. Biomed Chromatogr 16, 427-431.

Wender R, Brown AM, Fern R, Swanson RA, Farrell K \& Ransom BR (2000). Astrocytic glycogen influences axon function and survival during glucose deprivation in central white matter. J Neurosci 20, 6804-6810.

Winder WW, Yang HT, Jaussi AW \& Hopkins CR (1987). Epinephrine, glucose, and lactate infusion in exercising adrenodemedullated rats. J Appl Physiol 62, $1442-1447$.

\section{Author contributions}

Experiments were performed at the Laboratory of Exercise and Neuroendocrinology at the University of Tsukuba. All the listed authors contributed in the following ways: (1) Conception and design of the experiment; (2) Collection, analysis and/or interpretation of data; (3) Drafting the article or revising it critically for important intellectual content. All authors approved the final version. 


\section{Titles and legends for figures}

Fig. 1: Blood glucose and glycogen levels in muscles, the liver, and several brain loci immediately after treadmill running exercise or sedentary behavior for $\mathbf{1 2 0}$ min. (A) Blood glucose levels, (B) Muscle glycogen levels, (C) Liver glycogen levels, (D) Glycogen levels in eleven brain loci, and $(E)$ Reduction rate of brain glycogen. Data represent the mean $\pm \operatorname{SEM}\left(\mathrm{n}=5-11\right.$ rats). ${ }^{*}, p<0.05 ;{ }^{* * *}, p<0.0001$ compared to sedentary rats (unpaired $t$-test).

Fig. 2: Blood glucose and glycogen levels in muscles, the liver, and several brain loci, immediately after treadmill running exercise for 0 (Pre-Exercise), 30, 60, and 120 min. (A) Blood glucose levels, (B) Blood lactate levels, (C) Soleus glycogen levels (D) Plantaris glycogen levels (E) Liver glycogen levels (F) Cortex glycogen levels, $(G)$ Hippocampus glycogen levels, (H) Hypothalamus glycogen levels, (I) Cerebellum glycogen levels, and $(\mathrm{J})$ Medulla oblongata glycogen levels. Data represent the mean \pm SEM ( $\mathrm{n}=5-6$ rats). ${ }^{*}, p<0.05 ;{ }^{* *}, p<0.01 ;{ }^{* *}, p<0.0001$ compared to Pre-Exercise (Dunnett's post hoc test).

Fig. 3: Correlation between brain glycogen levels and blood glucose (A), brain glucose (B), and brain lactate (C) (Pearson's product-moment correlation test).

Fig. 4: Monoamines and their metabolites levels in the cortex immediately after treadmill running exercise for 0 (Pre-Exercise), 60, and $120 \mathrm{~min}$, and correlation between monoamine metabolites and glycogen levels in the cortex (Pearson's product-moment correlation test). Data represent the mean \pm SEM ( $n=4-6$ rats). (A) Noradrenaline (NA), (B) 5-hydroxytryptamine (5-HT), (C) Dopamine (DA), (D) Methoxyhydroxyphenylglycol (MHPG), (E) 5-hydroxyindoleacetic acid (5-HIAA) (F) 3,4-dihydroxyphenylacetic acid (DOPAC), ${ }^{*}, p<0.05 ;{ }^{* * *}, p<0.0001$ compared to Pre-Exercise, \#, $p<0.05$ compared to 60 min (Tucky's post hoc test). (G) Correlation between MHPG and glycogen levels, $(\mathrm{H})$ Correlation between 5-HIAA and glycogen levels, and (I) Correlation between DOPAC and glycogen levels. 
(A) Blood glucose

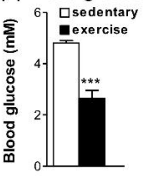

(B) Muscle glycogen

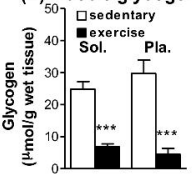

(C) Liver glycogen

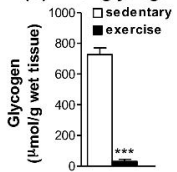

(D) Brain glycogen

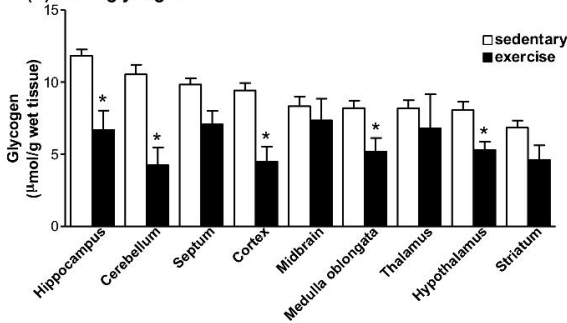

(E) Reduction rate of brain glycogen

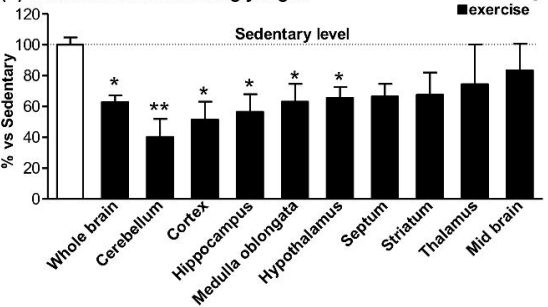

Fig. 1 
(A) Blood glucose

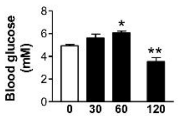

(B) Blood lactate

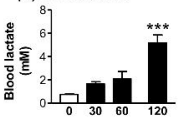

(C) Soleus glycogen

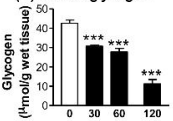

(D) Plantaris glycogen

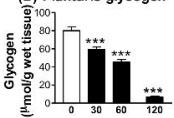

Running time (min)
(E) Liver glycogen

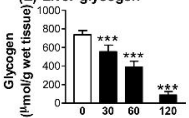

(F) Cortex glycogen

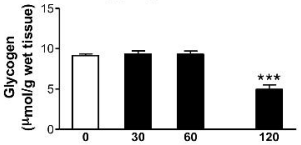

(G) Hippocampus glycogen

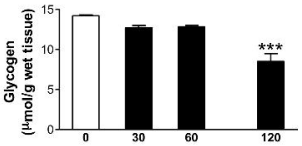

(I) Cerebellum glycogen

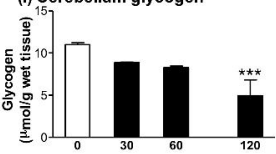

(H) Hypothalamus glycogen

(J) Medulla oblongata glycogen
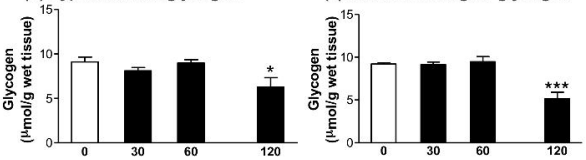

Running time (min)

Fig. 2 


\section{(A) Blood glucose vs. Brain glycogen}
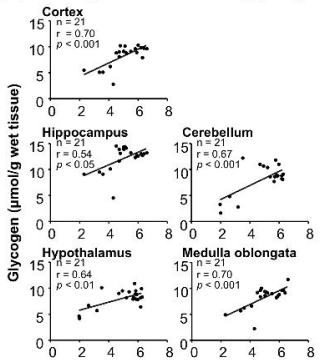

Blood glucose (mM)

(B) Brain glucose vs. Brain glycogen

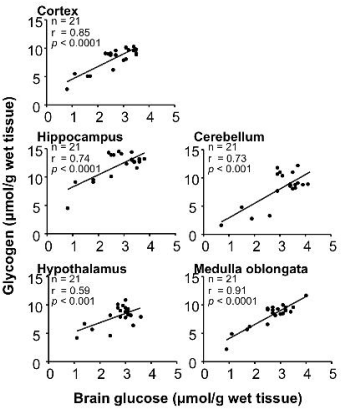

(C) Brain lactate vs. Brain glycogen Cortex

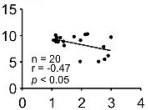

Hippocampus

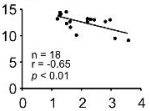

Hypothalamus

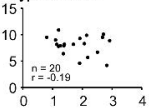

Cerebellum

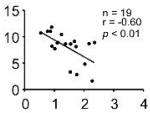

Medulla oblongata

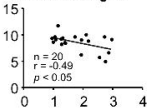

Lactate ( $\mu \mathrm{mol} / \mathrm{g}$ wet tissue) 


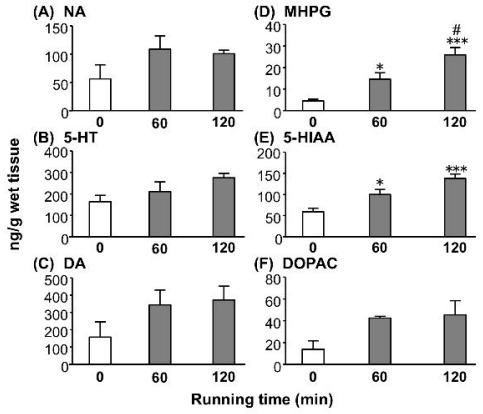

(G) MHPG vs. glycogen

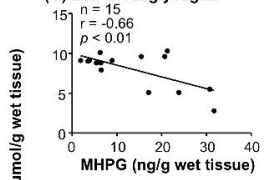

(H) 5-HIAA vs, glycogen

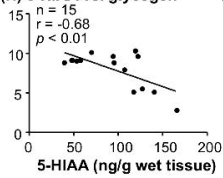

(I) DOPAC vs. glycogen

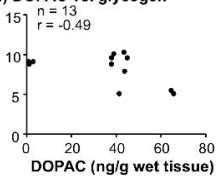

Fig. 4 